\title{
0 olhar por diferentes lentes: o photovoice enquanto método científico participativo
}

\author{
Daniel Meirinho
}

Artigo recebido em: 26/08/2016 Artigo aprovado em: 07/11/2017 


\title{
0 olhar por diferentes lentes: \\ o photovoice enquanto método científico participativo
}

\author{
The look between different lenses: \\ the photovoice as a participative scientific method
}

Daniel Meirinho*

Resumo: Este artigo intenciona promover uma reflexão teórica em torno da aplicabilidade e usos da fotografia participativa abordada pelo método Photovoice, contextualizando a importância da imagem fotográfica enquanto ferramenta metodológica em pesquisas sociais e colaborativas. Por fim, levantaremos um debate acerca dos beneficios e potenciais da metodologia Photovoice, assim como alguns dilemas, desafios e limitações da fotografia participativa como ferramenta de pesquisa-ação.

Palavras-chaves: Photovoice. Fotografia participativa. Metodologia visual. Pesquisa-ação.

Abstract: This article intends to promote a theoretical reflection on the applicability and uses of participatory photography approached by Photovoice method, contextualizing the photographic image importance as a methodological tool in social and collaborative researches. Finally, we will raise a debate about the Photovoice methodology benefits and potentials, as well as some dilemmas, challenges and limitations of participatory photography as action research tool.

* Daniel Meirinho é doutor em Ciências da Comunicação, na especialidade Comunicação e Ciências Sociais pela Faculdade de Ciências Sociais e Humanas, da Universidade Nova de Lisboa (FCSH/UNL). É pesquisador associado do Centro de Investigação Media e Jornalismo (CIMJ) e Professor Adjunto do Departamento de Comunicação Social da Universidade Federal do Rio Grande do Norte (UFRN). 
Keywords: Photovoice. Participatory photography. Visual methodology. Action research.

\section{A fotografia participativa como elemento metodológico da pesquisa-ação}

A fotografia há muito tempo tem sido utilizada com o intuito de documentar e chamar a atenção para as questões sociais. Tradicionalmente, as realidades sociais de contextos problemáticos são captadas por profissionais como documentaristas, jornalistas e fotógrafos. Neste sentido, o carácter profissional é que legitima e credibiliza a representação visual. Contudo, o universo representado nas imagens fotográficas é apresentado, muitas vezes, a partir do ponto de vista de um agente externo e suas escolhas são influenciadas pelos seus repertórios pessoais que podem não interagir com os ambientes sociais ou indivíduos fotografados.

Ao desconhecer uma determinada realidade, ou pelo simples fato de estar fora de um determinado contexto, o fotógrafo pode, ou não, distorcer a carga informativa que a imagem fotográfica possui. O recurso visual pode ser percebido como uma forma de conexão entre estranhos, criando caminhos que guiam para o desconhecido e o subjetivo. O carácter amplo do imaginário fotográfico alarga essa conexão. Para o observador, "as imagens podem funcionar como ponto de partida e de referências para discussões deste desconhecido e seu conteúdo literal pode, inclusive, ser lido dentro do cruzamento de fronteiras culturais." (COLLIER JUNIOR, 1987, p. 99).

Para os pesquisadores sociais, o modelo alarga as perspectivas analíticas das investigações e, para os participantes, os benefícios do uso de metodologias visuais colaborativas incluem a validação do repertório de vida e conhecimento local (SPIELMAN, 2001), novas perspetivas sobre si próprios e sua situação (MCINTYRE; 
THUSI, 2003), aumento da autoestima (EWALD, 2001; LYKES; BLANCHE; HAMBER, 2003), reforço a equidade de gênero (WILLIAMS; LYKES, 2003), reconhecimento e reflexão enquanto grupo (LYKES; BLANCHE; HAMBER, 2003) e defesa coletiva direcionada para a mudança social (MCALLISTER et al., 2005; WANG; REDWOOD-JONES, 2001).

Diferentes terminologias são utilizadas por distintos autores que descrevem este tipo de pesquisa-ação. Essas variam de imagebased research, denominada por Prosser (1998), a métodos visuais (BANKS, 2001) ou metodologias visuais (ROSE, 2001). A recolha e análise de dados baseados na imagem fotográfica participativa têm sido notavelmente utilizadas em estudos antropológicos e etnográficos. Contudo, são geralmente complementares a outros métodos mais tradicionais (MORROW, 2001; PROSSER, 1998). Estudos demonstram o crescimento do uso da fotografia como ferramenta metodológica (PROSSER; SCHWARTZ, 1998). No entanto, as imagens fotográficas ainda são subutilizadas (BOLTON; POLE; MIZEN, 2001) e ainda são escassos os casos que aparecem como único objeto central de análise. Um largo corpo de pesquisa, numa variedade de áreas e contextos, existe e indica a eficácia desta metodologia (WANG et al., 2004).

A fotografia participativa tem vindo a se tornar uma importante subdivisão das Participatory Action Researches (PAR) (KHANLOU; PETER, 2005). Isso porque passa a integrar à pesquisa empírica um processo de aprendizagem colaborativa, sedimentando a possibilidade de beneficiar grupos excluídos e minorias que estão fora da discussão e formulação das políticas públicas que os afetam (GREENWOOD; LEVIN, 2007). A metodologia incorpora um modelo participativo dos grupos estudados no duplo objetivo de ação e pesquisa, no sentido de obter resultados em ambas as vertentes. As ações voltadas a mudanças e transformações pessoais e coletivas num determinado contexto possuem a finalidade de ampliar o entendimento, por parte dos pesquisadores, das 
relações comunitárias e entre os seus membros (DICK, 2000). Especificamente o método, quando bem implementado, possui o poder de ativar os benefícios práticos centrados no conhecimento experiencial para a cooperação na pesquisa (HERON, 1996). Harper (2003), quando reflete sobre pesquisas que usam a fotoetnografia, ressaltou que a câmera pode "representar uma realidade na mesma proporção que um olho enxerga, apenas ligeiramente mais estreito que a visão normal.” (HARPER, 2003, p. 258).

A imagem pode revelar aspectos e perspetivas que poderiam não ser aparentemente tão visíveis em outras metodologias aplicadas a contextos sociais. Sobre os benefícios e vantagens para os pesquisadores e participantes de uma pesquisa participativa visual, Esther Prins (2010) salientou a amplitude que a imagem fotográfica pode oferecer:

Os participantes representam visualmente suas experiências (apresentando saberes), enquanto também aprendem a tirar fotografias (saber prático), interagindo de diferentes maneiras com pessoas distintas (saber experimental) e desenvolvendo novas compreensões conceituais (saber proposicional) (PRINS, 2010, p. 428).

As pesquisas através de metodologias visuais passam a ser ferramentas poderosas para conjurar conhecimento em vários níveis. Ao longo dos anos, cientistas sociais têm questionado a neutralidade dos dados contidos nas fotografias. Contudo, Prosser e Schwartz (1998, p. 116) afirmaram que "manter a investigação baseada em imagem ainda fornece um valioso contributo para as Ciências Sociais Aplicadas porque as imagens fotográficas podem fornecer dados e conhecimento que não podem ser traduzidos em outras formas de comunicação." A possibilidade de manipular os resultados e a subjetividade podem ser elementos também presentes em outros modelos metodológicos. Neste sentido, a conduta ética e o olhar analítico do pesquisador são fundamentais para a redução da distorção dos dados. Mesmo sabendo que não existe uma análise 
neutra.

Defensor entusiasta do uso da fotografia em pesquisas científicas, Rob Walker (1993) acredita que o meio visual pode oferecer uma voz qualitativa única. Argumenta que as imagens visuais não fornecem ao observador apenas uma visão da vida de outras pessoas, mas também podem dar um "vislumbre das maneiras como os indivíduos criam significado sobre as suas próprias vidas" (WALKER,1993, p. 82).

Iremos nos alicerçar especificamente nos conceitos da metodologia participativa conhecida como Photovoice, que será aprofundada nos próximos tópicos. Neste método, a fotografia é o instrumento para "representação de perspectivas daqueles que levam uma vida diferente dos meios que tradicionalmente possuem o controle das imagens do mundo" (WANG, 2006, p. 154). A fotografia na pesquisa participativa serve como uma alternativa ao registo escrito, o qual, por si só, promove a inclusão dos participantes como informantes e até mesmo como pesquisadores válidos, no caso das pesquisas colaborativas. Encará-los como competentes para o manuseamento de equipamentos de registo em fotografia é uma atitude indispensável para poderem documentar e tornar visíveis as suas representações acerca do mundo que os rodeia.

Ao pensarmos em grupos sociais, especialmente em situação de vulnerabilidade e exclusão social, o recurso passa a ser uma estratégia que supera as suas limitações de expressão por forma textual. Desta forma é proporcionada uma ferramenta adicional que facilita aos envolvidos um modelo diferente para articulação das suas experiências pessoais e, desta forma, contribui para uma reflexão mais detalhada das suas relações e perfis identitários. As problemáticas relativas à alfabetização deficiente ou da língua, nestes contextos, são características que podem criar relativas dificuldades no processo de protagonismo e participação (ALLEN, 2002).

A fotografia pode ser vista nos estudos de pesquisa-ação 
participativos como um instrumento catalisador de mudanças, justificado através da dupla função que a imagem fotográfica pode assumir: como expressão criativa visual ou como um meio de retratação de realidades e contextos. Neste sentido, a imagem fotográfica pode ser o instrumento que fornece aos pesquisadores sociais oportunidade de aceder distintas realidades, perspectivas, comunicar e sensibilizar participantes para as questões sociais que os rodeiam. Seu relativo baixo custo e facilidade de divulgação possibilitaram o compartilhamento e potencialização de diálogos, facilitando que as discussões ultrapassassem as barreiras culturais e linguísticas de cada contexto.

\section{Photovoice: uma ferramenta participativa para o desenvolvimento local}

No início dos anos 90, Caroline Wang, professora e pesquisadora da Escola de Saúde Pública da Universidade de Michigan, e Mary Ann Burris, investigadora associada da Escola de Estudos Orientais e Africanos da Universidade de Londres, desenvolveram, em parceria, uma abordagem teórica e metodológica para projetos de pesquisa-ação participativos que chamaram de Photovoice (WANG, 1999). O método se propõe a inserir no processo de pesquisa atividades de base comunitária com a finalidade de capacitar em conjunto membros de grupos sociais em situação de exclusão no intuito de "identificar, representar e reforçar os recursos das suas comunidades através de técnicas e representações fotográficas." (WANG; BURRIS, 1997, p. 369). A fotografia passa a ser um suporte e ferramenta de trabalho "que serve como instrumento para criar relações, informar e organizar indivíduos da comunidade, permitindo-lhes dar prioridade às suas preocupações e discutir seus problemas e soluções coletivamente, através dos enquadramentos visuais." (WANG; BURRIS 1997, p. $370)$. 
Com base no conceito criado pelas autoras, anteriormente chamado de Photo Novella, a "voz" no Photovoice é compreendida como um acrónimo para Voicing Our Individual and Collective Experience ${ }^{1}$. Este é usado durante as discussões orientadas para estimular os participantes a "refletirem sobre suas próprias condições de vida, mas também no sentido de partilhar as suas experiências" (WITH et al., 2009, p. 6). De acordo com o Practical Guide to Photovoice (2009), a ideia é fundamentada na utilização pelos indivíduos de diferentes formas de imagens e palavras para expressar o que necessitam, o que se preocupam, o que têm medo, estimam e sonham (WITH et al., 2009). Uma das finalidades do método é o "acesso aos mundos das outras pessoas para que esses mundos se tornem acessíveis" (BOOTH; BOOTH, 2003, p. 431), tanto para outros grupos de indivíduos, quanto para as pesquisas sociais.

O Photovoice foi criado com base na promoção da saúde pública, desenvolvimento comunitário e educação; suas primeiras aplicações compunham essa tríade (WANG et al., 1998; WANG; BURRIS, 1997; WANG; BURRIS; XIANG, 1996). Só com o passar dos anos, outras questões mais voltadas ao campo social, cultural e identitário foram acrescentadas aos estudos que utilizavam este método. Com a ampliação deste campo de atuação, a metodologia passou a ser frequentemente aplicada nas populações minoritárias e em contextos de vulnerabilidade e exclusão, propondo-se a apontar caminhos e pontos de reflexão sobre as circunstâncias que envolvem seus membros na esperança de melhorias futuras, num processo de incidência politica e advocacy (DAW, 2011; GOODHART et al., 2006; NEWMAN, 2010).

O método é fortemente influenciado pelos resultados dos estudos community-based participatory research dos investigadores Nina Wallerstein e Bernstein (1988). A fundamentação do processo é construída a partir dos princípios inerentes à fotografia documental, à teoria feminista e aos estudos da educação para a consciência

1 "Expressando nossas experiências individuais e coletivas" (tradução livre). 
crítica, do pedagogo Paulo Freire (WALLERSTEIN; BERNSTEIN, 1988; WANG; BURRIS, 1994, 1997). A primeira experiência de aplicação da metodologia foi realizada em 1994 num projeto de saúde sexual e reprodutiva com mulheres da província de Yunnan, na China. O objetivo do estudo, chamado Empowerment through Photo Novella: Portraits of Participation (WANG; BURRIS, 1994), foi utilizar as representações visuais para influenciar as políticas e programas que afetavam as mulheres do contexto rural (WANG; BURRIS, 1997).

A proposta metodológica, no campo dos estudos de saúde pública, aponta casos de sucesso em diferentes contextos e populações (WANG, 1999; WANG; BURRIS, 1997; WANG; CASH; POWERS, 2000). Estes vão desde grupos de indivíduos sem-teto (REMILLARD, 2012), a agentes comunitários de saúde, e professores de zonas rurais na África do Sul (LANGE; MITCHELL; STUART, 2008), sobreviventes de lesão cerebral (LORENZ, 2010). Tem sido aplicado ainda com grupos de organizações feministas e de direitos das mulheres (LYKES, 2001; MCINTYRE; THUSI, 2003).

Através do interesse de autorrepresentação comunitária, o Photovoice se propõe a trazer perspetivas diferentes das que os meios sociais e mediáticos tradicionais desenvolvem. Os participantes são envolvidos nos processos de decisões de suas próprias políticas (WANG; BURRIS, 1997), neste sentido, sua estratégia passa a ser bastante eficaz na resposta a questões e identificação de problemáticas levantadas à luz da sua própria autorrepresentação.

\section{Benefícios e potencial do Photovoice: Contextualizando o método}

A corrente de autores que estuda as metodologias participativas visuais descreve o Photovoice como uma estratégia de pesquisa altamente flexível e capaz de ser "adaptada a objetivos 
específicos participativos, com diferentes grupos e comunidades na reflexão e formulação de políticas públicas" (WANG; BURRIS 1997). Práticas e formas de compreensão do mundo através da fotografia documental, ação social e consciência crítica são pontos que diferenciam esta de algumas metodologias mais tradicionais (WANG, 1999; WANG; BURRIS, 1994, 1997). A mistura entre pensamentos teóricos mais flexíveis e práticas inovadoras resulta numa abordagem que desafia modelos usuais de pesquisa científica. Uma breve discussão sobre fusão de diferentes teorias e práticas vai nos ajudar a compreender melhor o Photovoice.

Para Wang (1999), o Photovoice se diferencia de outras formas de representação social visual porque a câmera está nas mãos de membros da comunidade, em vez de na posse de um sujeito externo à realidade local. Os participantes do processo aprendem a operar câmeras fotográficas e são convidados a registar os pontos positivos e negativos das suas comunidades. As autoras apontam que os objetivos específicos do método incluem criar competências nos participantes com o propósito de utilizar imagens fotográficas para documentar e refletir sobre as necessidades e os recursos comunitários. A partir dos pontos de vista e reflexões dos indivíduos envolvidos sobre as fotografias que produziram, é construído um ambiente dialógico acerca de quais são as questões importantes e as preocupações de cada participante (WANG; BURRIS, 1994, 1997; WANG; BURRIS; XIANG, 1996).

Os participantes são percebidos como sujeitos ativos capazes de compreender as suas questões comunitárias através de uma partilha mútua de experiências que os transforma em possíveis agentes da mudança pessoal e social (FREIRE, 1963). Wang descreveu o seu método como um processo de pesquisaação participativo que confia câmeras fotográficas "àqueles que raramente têm acesso e tomam decisões sobre suas vidas." (WANG; BURRIS; XIANG, 1996, p. 1391). De acordo com os guias e manuais práticos de intervenção (BLACKMAN, 2007; WITH et al., 2009) o método enfatiza três ideias bases: (1) as experiências 
subjetivas vividas pelos indivíduos, (2) a representação individual de sua própria realidade e (3) o empoderamento de grupos excluídos, sub-representados e silenciados.

Carole Wang (2006) aconselha uma estratégia fundamentada em nove etapas para mobilizar a ação comunitária através do uso do Photovoice. Wang (2006) sugeriu que as nove fases fossem desenvolvidas de tal ordem:

1. Identificar os decisores politicos na comunidade com algum perfil de liderança comunitária. É levantada a questão: Quem tem o poder de tomar decisões que podem melhorar a situação? (WANG, 2006). Podem ser incluídos membros da comunidade e atores sociais influentes para a concretização da disseminação dos resultados e vontade política do grupo.

2. Recrutar um grupo de participantes para o Photovoice. Para permitir a facilidade prática e uma discussão aprofundada, as autoras recomendam entre sete a dez participantes com igualdade de gênero. Estes podem ser mobilizados através de distintos níveis de escolaridade, grupos pertencentes a instituições religiosas, de preferência já estruturados e organizados. Além disso, é fundamental identificar projetos e parceiros que possam fazer parte da rede, proporcionando uma mobilização comunitária mais eficaz. "As etapas 1 e 2 não precisam seguir necessariamente esta ordem. Podem ser intercambiáveis sem causar problemas futuros" (WANG, 2006, p. 149-150).

3. Introduzir a metodologia Photovoice aos participantes e facilitar uma discussão de grupo sobre imagem, poder e ética. $\mathrm{O}$ primeiro encontro deve ser iniciado pelas regras de funcionamento da intervenção, criadas em conjunto com os participantes. As questões éticas devem vir logo em seguida para um entendimento sobre a responsabilidade e autoridade que lhes serão atribuídas enquanto fotógrafos empunhando a câmera. $\mathrm{O}$ respeito e agradecimento pela colaboração devem ser transversais ao processo em todos os momentos de captação fotográfica. Desta forma minimizam os riscos potenciais para o bem-estar dos participantes e das pessoas 
fotografadas. Para oferecer suporte aos facilitadores e envolvidos, Wang (2006) sugere a discussão de questões que incluem: Qual a forma aceitável para se aproximar de alguém para fotografá-lo? Deve-se tirar fotografias de pessoas sem o consentimento delas? Em que momentos não gostarias que lhe tirassem uma fotografia? A quem poderias mostrar suas imagens fotográficas e quais os impactos que causaria?

4. Obter o consentimento informado. Esta é uma etapa sugerida por alguns autores (WANG; BURRIS, 1997) e criticada por outros (MERRICK; MEJIA, 2010; VAUGHAN, 2011) por ser uma característica limitadora à participação e ao envolvimento dos grupos sociais envolvidos. Contudo todos aconselham que nas primeiras sessões sejam enfatizadas questões como segurança, autoridade e responsabilidade que são consequentes ao uso de uma câmera. Os consentimentos informados em alguns casos são solicitados e podem ser por escrito, com a inclusão de um resumo da proposta e os potenciais riscos de participação. As fotografias não podem ser identificadas sem a devida autorização (WANG; REDWOOD-JONES, 2001).

5. Identificar um ou mais temas para as fotografias. Os participantes podem desenvolver debates sobre as temáticas em conjunto e assim determinar o que cada um deseja fotografar. Ou, dado um tema específico, podem discutir maneiras em que esses tópicos possam ser representados. Após a captação fotográfica são realizadas discussões e reflexões acerca das representações visuais e das questões levantadas nas imagens.

6. Distribuir as câmeras para os participantes e rever como usá-las. Uma questão pode ser colocada na idealização da proposta. Que tipo de câmera deve ser usada? Wang e Burris (1997) apontam para pelo menos quatro diferentes tipos de dispositivos já utilizados em outros projetos Photovoice: câmeras monoreflex, de visor direto, descartáveis, de médio formato (Holga), digitais e acopladas aos celulares. As escolhas podem ser guiadas pelas preferências dos facilitadores e dos participantes ou pelos recursos disponíveis 
(WANG, 2006), podendo ser incluídos outros dispositivos de captação fotográfica.

7. Dar tempo aos participantes para tirar as fotografias. Os participantes devem determinar um tempo para estarem de posse das câmeras para retornarem com as fotografias que se propuseram a captar. Em seguida, mais uma vez se reúnem para discutir suas fotografias.

8. Promover reuniões para discutir as fotografias e identificar os recursos e problemáticas comunitárias. Três estágios fornecem as bases de análise das imagens captadas que são chamadas de seleção, contextualização e codificação. Em primeiro lugar, cada participante seleciona algumas fotografias que melhor refletem as suas preocupações e identificam os recursos comunitários. A abordagem participativa é firmada nesta primeira etapa.

Num segundo momento, os participantes contextualizam as histórias contidas nas imagens. A abordagem participativa também gera a segunda etapa centrada no processo de discussão em grupo, sugerido pela sigla $v o z$, expressa nas experiências individuais e coletivas. Um quadro de diálogo crítico é indicado através de questões como: $\mathrm{O}$ que vês? $\mathrm{O}$ que realmente está a acontecer na imagem? Como isso relaciona-se com as nossas vidas? Porque esta situação o preocupa? O que podemos fazer sobre isso?

O terceiro momento de análise das imagens é fundamentado na codificação e identificação das questões, temas e teorias que emergem nas fotografias. Nesta etapa, é facultado aos participantes reconhecerem três tipos de dimensões que surgem a partir do processo de diálogo: questões, temas ou teorias. Os participantes podem codificar os problemas quando as preocupações são alvos pragmáticos, imediatos e tangíveis para a ação social. Esta é a aplicação mais direta da análise. Essas três fases devem ser executadas para cada rodada de fotografias tiradas pelos envolvidos na pesquisa (BLACKMAN, 2007). A quantidade destes momentos depende de fatores como as preferências dos pesquisadores e participantes, orçamento, disponibilidade e outras considerações 
de ordem prática e logística.

9. A última etapa para a mobilização e uso do método Photovoice é fundamentada no planejamento partilhado dos formatos de disseminação das imagens fotográficas e histórias produzidas. A mostra das imagens pode ser feita através de inúmeros meios e estratégias com o objetivo de disseminar os trabalhos, histórias e recomendações aos familiares, membros da comunidade, lideranças e decisores políticos locais.

Baseando-se em Foucault, Esther Prins (2010) ressaltou que a metodologia ativa e valida "saberes subjugados em ressonância com condições prévias de diálogo" (2010, p. 439). Foster-Fishman et al. (2005), Hodgetts, Chamberlain e Radley (2007) e Castleden e Garvin (2008) declaram que o ato de retratar e de buscar assuntos e temas para fotografar pode significar o reenquadramento de assuntos comuns pelos participantes, alinhado ao processo de conscientização crítica. "É um método que "leva a sério os participantes como conhecedores de seus contextos" (GUILLEMIN; DREW, 2010, p. 178). Prins (2010, p. 427) vai além na análise de algumas iniciativas de uso do método Photovoice e ressalta que "tais projetos possuem a peculiaridade e potencial para subverter as relações hierárquicas entre o pesquisador e participantes, permitindo pessoas comuns investigarem e representarem suas próprias vidas."

Numa análise detalhada do impacto sobre os participantes, Foster-Fishman et al. (2005) identificou três principais resultados: o aumento da autocompetência; o aperfeiçoamento da consciência crítica do meio em que vivem; e o cultivo de recursos para a ação social e possivelmente política (incluindo a construção de relações entre os participantes e os tomadores de decisão). Estes resultados promissores sugerem o Photovoice como uma ferramenta útil e adequada em estudos participativos em que o indivíduo é incentivado a tomar as suas próprias decisões (FOSTER-FISHMAN et at., 2005). 


\section{Desafios, dilemas e constrangimentos do método Photovoice}

O método Photovoice tem sido usado em diferentes contextos e populações com o objetivo de promover competências nos seus participantes para que possam representar suas realidades através de um formato de expressão visual. Como dito, a técnica não necessita de equipamentos e formações complexas e como tal pode ser uma ferramenta poderosa e eficaz em contextos sociais, possibilitando o desenvolvimento de habilidades visuais. Por estes benefícios, a fotografia participativa vem a se firmar como um interessante instrumento de trabalho para pesquisadores sociais em estudos com grupos de exclusão social, econômica e/ou cultural (SINGHAL et al., 2007).

Embora a literatura sobre a temática enumere vantagens referentes ao método fotográfico participativo, se faz necessário precavermos, antes mesmo da execução de um projeto de pesquisa colaborativo, alguns desafios e limitações apontados por estudos anteriores acerca da aplicabilidade do Photovoice (LYKES; BLANCHE; HAMBER, 2003; MCINTYRE; LYKES, 2004; WANG; REDWOOD-JONES, 2001).

Diversos estudos nos fornecem experiências, resultados e erros que demonstram que a fotografia participativa pode oferecer resultados inequívocos como qualquer outro instrumento de análise social (GALLO, 2001; MCALLISTER et al., 2005; SPIELMAN, 2001). Aintervenção visual, quandonão implementada corretamente, pode subestimar o conceito de participação e perpetuar ainda mais o binário estabelecido entre 'silenciar' e o 'dar voz' (LYKES; BLANCHE; HAMBER, 2003). Uma gama de desafios e limitações para o método Photovoice é levantada pelos mesmos autores que trabalham a sua aplicabilidade. Em seus primeiros trabalhos, Wang e Burris (1997) sublinharam a natureza política e da ação participativa para documentar questões comunitárias através da 
fotografia. Tal como acontece com qualquer ato interventivo social, as autoras apontaram a possibilidade dos riscos potenciais para os participantes e aqueles a quem são fotografados, tomando em conta os riscos aliados a exposição de ideais e análises individuais de contextos particulares.

Os projetos que utilizam o método Photovoice necessitam refletir sobre alguns dilemas éticos como a invasão de privacidade através da divulgação de fatos embaraçosos sobre indivíduos (WANG; BURRIS, 1997). Neste sentido, os cuidados devem ser redobrados pois uma imagem pode representar falsamente uma realidade pelo seu produtor para favorecimento positivo, e também negativo, de uma causa comunitária (WANG; REDWOOD-JONES, 2001). Para atenuar esses riscos, é sugerido que "as atividades do Photovoice devam ser iniciadas com uma discussão ética e sobre o poder que a fotografia pode vir a ter" (LYKES et al., 1999, p. 218). É necessário que os pesquisadores estejam atentos, se antecipem e prevejam a possibilidade de controvérsia e manipulação das imagens e dos discursos (EWALD, 2001).

Um outro ponto específico que merece atenção é a segurança do participante no processo de captação fotográfica, quanto aos riscos associados a fotografar atos ilegais ou indesejáveis (particularmente em relação ao uso e venda de drogas, trabalho sexual, armas, entre outros). Como apontou Vaughan (2011) "tais temas levantam a discussão de casos em que os participantes podem potencialmente produzir 'provas' fotográficas que podem ser usadas contra aqueles que foram fotografados ou contra os próprios fotógrafos" (VAUGHAN, 2011, p. 100). Apesar de alguns participantes não perceberem ou identificarem situações constrangedoras ou até perigosas, é fundamental e de responsabilidade do pesquisador incentivar uma reflexão sobre o que é ou será captado. Como ocorre com os métodos de pesquisa tais como entrevistas e discussões em grupo, os participantes também podem evitar levantar temáticas específicas com o intuito de proteger os investigados de uma exposição indesejada e de questões sensíveis que possam causar- 
lhes desconforto.

Ao analisarmos alguns exemplos de pesquisas que utilizaram o método visual participativo, encontramos recomendações como as de Williams e Lykes (2003) que detalham algumas problemáticas encontradas em relação ao gênero. Os autores comentaram sobre a relutância das mulheres em alguns contextos específicos em participarem de forma mais efetiva e fotografarem, devido a uma estrutura machista em que o homem possui relações de poder bastante fortalecidas e de influencia nos grupos de mulheres. Para Ewald (2001) os fotógrafos-participantes podem encontrar ainda hostilidade ou descobrir que suas fotografias criaram controvérsia entre o grupo e na comunidade (EWALD, 2001; WILLIAMS; LYKES, 2003). Essas percepções revelaram que tirar e exibir fotografias, incorporando histórias locais nas imagens, pode provocar um distúrbio na forma como os indivíduos se relacionam entre si e com o mundo exterior devido ao mergulho que é proporcionado pela captação, análise e reflexão das imagens.

Em alguns casos o desejo de "dar voz" pode levar a uma aceitação acrítica das representações visuais (PIPER; FRANKHAM, 2007), sem perceber que o ato de "dar a voz" já é um posicionamento de poder do pesquisador sobre o participante, em muitos casos em situação de risco, pois este possui a voz especializada e detém as formas de poder, ofertando-as. Outra limitação é que não foi fotografado pode deixar de ser analisado e refletido (HODGETTS; CHAMBERLAIN; RADLEY, 2007). Em contraste, é necessário que a equipe de pesquisa reconheça e deixe que os participantes escolham livremente as histórias que desejam contar sobre si mesmos e sobre as suas comunidades. Esta liberdade de escolha passa a ser um elemento fundamental, pois determina como gostariam de ser vistos através do processo de decisão e não como os facilitadores da proposta queriam que eles fossem vistos.

A ausência ou o silêncio podem ser encontrados caso as temáticas direcionem os envolvidos na pesquisa para questões quase "infotografáveis". Wang e Pies (2004) destacaram que os 
tópicos podem ser suprimidos pelos participantes por duas formas. Ou porque eles não são importantes para suas vidas, ou porque o tema é difícil de se fotografar. Mesmo assim os envolvidos podem demonstrar uma grande criatividade para ilustrar questões delicadas e subjetivas de retratar ou na captura de questões sensíveis de forma compreensível (SINGHAL et al., 2007).

Os pesquisadores devem ter em conta que perdas, danos e roubos de equipamentos são possíveis riscos e que as considerações de ordem ética e consentimentos podem restringir a quantidade de representações fotográficas dos indivíduos que participam do universo comunitário. "Quando pensamos nos ganhos objetivos para a comunidade e as expectativas que um projeto deste gera, os resultados podem também não ser tão significativos para os seus membros quanto se espera." (WITH et al., 2009, p. 18), o que pode gerar frustrações do que se propõe um projeto e do que se alcança concretamente.

Consideramos a opinião de Pini (2001, p. 24) quando mencionou que "os pesquisadores devem ter a percepção que tirar fotografias ou fazer vídeos não garante aos participantes liberdade do controle de influência para tomar suas próprias decisões e para contar qualquer história sobre eles próprios." Deve-se ter em conta que um modelo de pesquisa interventivo, mesmo sem intenção, inevitavelmente os objetivos podem vir a moldar subtilmente as ações e escolhas dos participantes para o benefício do estudo. Esse cuidado teve que estar permanente em todo o desenvolvimento da pesquisa.

O envolvimento num projeto de fotografia participativa pode levar a algum protagonismo, mas torna-se fundamental ter em conta os constrangimentos. Tal situação pode reverter as relações de poder e promover uma desmobilização ao invés de uma ordenação para algumas transformações. O planejamento das atividades e o reconhecimento do campo evitam alguns dilemas, contudo, mesmo previstos muitos ambientes sociais e relações não podem ser controlados. 


\section{Notas Conclusivas}

O que este artigo apresenta é que ainda há muito a ser explorado relativamente às diferenças importantes na forma como o Photovoice pode ser mais eficazmente adaptado e usado em diversos contextos e públicos participantes. A aplicabilidade de projetos de pesquisa social que utiliza a fotografia participativa expressa uma conexão legítima com o desenvolvimento de uma consciência crítica nos envolvidos, tal como aludiu Paulo Freire, através de um modelo de aprendizagem dialógico e fundamentado na experiência dos participantes. Para Singhal (2004, p. 5), "Freire acreditava firmemente que elementos visuais criativos, especialmente se fossem expressados pelos indivíduos a desempenhar um papel chave na reflexão sobre suas próprias experiências, clarificação, articulação dos descontentamentos e a elaboração de soluções para uma ação social".

A imagem fotográfica pode ser compreendida pelas ciências sociais e humanas como uma efetiva ferramenta de pesquisa além de um recurso criativo que revela muito sobre as perspectivas individuais dos participantes. O uso livre do dispositivo fotográfico para a construção de um discurso visual demonstra-se que existem padrões e escolhas que refletem os seus perfis identitários e repertório cultural dos envolvidos do processo de pesquisa. Ao compreendermos a câmara enquanto uma janela através da qual o sujeito observa o mundo ao seu redor, esta pode ser entendida também como um espelho que reflete as suas posições perante o universo que observa e retrata em suas fotografias.

Pesquisas-acão que utilizam metodologias participativas visuais como o Photovoice demonstram como a narrativa pessoal e a experiência dos grupos envolvidos podem compor um quadro crítico para debater os tópicos e problemas que os afetavam. A comunicação visual é compreendida como uma forma sistemática para os participantes contarem suas próprias histórias, documentar e dar voz às suas ideias e interesses, sem as limitações de análises 
cruzadas dos agentes externos aos contextos sociais. Isso demonstra como a imagem fotográfica pode ser um instrumento pedagógico a ser utilizado nos processos de aprendizagens formais, na escola, e lúdicos, reforçando a literacia através da fotografia (EWALD, 2001), onde currículos educacionais poderiam incorporar a componente visual como pedagogia de confronto que provocaria os alunos a refletirem o conhecimento a partir de suas próprias constituições de saber e experiências de aprendizagem, tal como propôs Washabaugh (2008) sobre a literacia visual.

Projetos que utilizam o Photovoice, bem como outras metodologias visuais participativas, apresentam um valioso ponto de partida para traçar diferentes e dinâmicas estratégias de investigação com grupos sociais específicos através dos seus perfis identitários. Contudo, são necessários que sejam desenvolvidos mais estudos participativos visuais. Só assim a investigação académica colaborativa não só possibilitará novas perspectivas e olhares dos investigados sobre as suas realidades, como engajará os participantes a refletirem criticamente às preocupações que envolvem suas vidas.

\section{Referências}

ALLEN, Deborah. Research involving vulnerable young people: a discussion of ethical and methodological concerns. Drugs: Education, Prevention and Policy, Abingdon, v. 9, n. 2, p. 27583, 2002.

BANKS, Marcus. Visual methods in social research. Londres: Sage, 2001. 
BLACKMAN, Anna. The photovoice manual a guide to designing and running participatory photography projects. Londres: Photovoice, 2007.

BOLTON, Angela; POLE, Christopher J.; MIZEN, Phil. Picture this: researching child workers. Sociology, Oxford, v. 35, n. 2, p. $501-518,2001$.

BOOTH, Tim; BOOTH, Wendy. In the frame: photovoice and mothers with learning difficulties. Disability \& Society, Oxford, v. 18, n. 4, p. 431-442, 2003.

CASTLEDEN, Heather; GARVIN, Theresa. Modifying photovoice for community-based participatory Indigenous research. Social Science \& Medicine, Oxford, v. 66, n. 6, p. 1393-1405, 2008.

COLLIER JUNIOR, John. Visual anthropology's contributions to the field of anthropology. Visual Anthropology, New York, v. 1, n. 2, p. 37-46, 1987.

DAW, Matt. See it our way: participatory photography as a tool for advocacy. Londres: Photovoice, 2011.

LANGE, Naydene de; MITCHELL, Claudia; STUART, Jean. 
Putting people in the picture: visual methodologies for social change. Amsterdão: Sense Publishers, 2008.

DICK, B. A beginner's guide to action research. 2000. Disponível em: $\quad<$ http://www.scu.edu.au/schools/gcm/ar/arp/guide.html $>$. Acesso em: 30 mar. 2018.

EWALD, Wendy. I wanna take me a picture: teaching photography and writing to children. Boston: Beacon Press, 2001.

FOSTER-FISHMAN, Pennie et al. Using methods that matter: The impact of reflection, dialogue, and voice. American Journal of Community Psychology, Washington, v. 36, n. 3-4, p. 275-291, 2005.

FREIRE, Paulo. Conscientização: teoria e prática da libertação : uma introdução ao pensamento de Paulo Freire. São Paulo: Cortez \& Morales, 1963.

GALLO, Melina L. Immigrant workers' Journeys through a New Culture: exploring the transformative learning possibilities of photography. Studies in the Education of Adults, Leicester, v. 33, n. 2, p. 109-117, 2001. 
GOODHART, Fern Walter et al. A view through a different lens: photovoice as a tool for student advocacy. Journal of American College Health, Washington, v. 55, n. 1, p. 53-56, 2006.

GREENWOOD, Davydd James; LEVIN, Morten. Introduction to action research: social research for social change. California: Sage Publications, 2007.

GUILLEMIN, Marilys; DREW, Sarah. Questions of process in participant-generated visual methodologies. Visual Studies, v. 25, n. 2, p. 175-188, 2010.

HARPER, Douglas. Framing photographic ethnography: a case study. Ethnography, London, v. 4, n. 2, p. 241-266, 2003.

HERON, John. Co-operative inquiry: research into the human condition. Londres: Sage Publications, 1996.

HODGETTS, Darrin; CHAMBERLAIN, Kerry; RADLEY, Alan. Considering photographs never taken during photo-production projects. Qualitative Research in Psychology, London, v. 4, n. 4, p. 263-280, 2007.

KHANLOU, Nazilla; PETER, Elizabeth. Participatory action 
research: considerations for ethical review. Social Science \& Medicine, Oxford, v. 60, n. 10, p. 2333-2340, 2005.

LORENZ, Laura S. Visual metaphors of living with brain injury: exploring and communicating lived experience with an invisible injury. Visual Studies, v. 25, n. 3, p. 210-223, 2010.

LYKES, M. Brinton. Creative arts and photography in participatory action research in Guatemala. In: REASON, Peter; BRADBURY, Hilary (Ed.). Handbook of action research. Thousand Oaks: Sage, 2001. p. 363-371.

LYKES, M. Brinton; BLANCHE, Martim Terre.; HAMBER, Brandon. Narrating Survival and Change in Guatemala and South Africa: the politics of representation and a liberatory community psychology. American Journal of Community Psychology, Washington, v. 31, n. 1, p. 79-90, 2003.

LYKES, M. B. et al. Telling stories - rethreading lives: Community education, women's development and social change among the Maya Ixil. International Journal of Leadership in Education: Theory and Practice, v. 2, n. 3, p. 207-227, 1999. 
MCALLISTER, Carol L. et al. Come and take a walk": listening to early head start parents on school-readiness as a matter of child, family, and community health. American Journal of Public Health, New York, v. 95, n. 4, p. 617-625, 2005.

MCINTYRE, Angela; THUSI, Thokozani. Children and youth in Sierra Leone's peace-building process. African Security Review, v. 12, n. 2 , p. $73-80,2003$.

MCINTYRE, A.; Lykes, M. B. Weaving words and pictures in/ through feminist participatory action research. In: BRYDONMILLER, M.; MAGUIRE, P.; MCINTYRE, A. (orgs.). Traveling companions: Feminism, teaching, and action research. Westport, CT: Praeger, 2004.

MERRICK, Meg; MEJIA, Angie. Photovoice as authentic civic engagement: lessons learned in one immigrant community. São Francisco, California: Institute of Portland Metropolitan Studies Publications, 2010. Paper 138.

MORROW, Noreen Mac. Knowledge management: an introduction. Annual Review of Information Science and Technology, White Plains, v. 35, p. 381-422, 2001a. 
NEWMAN, Susan D. Evidence-Based advocacy: using photovoice to identify barriers and facilitators to community participation after spinal cord injury. Rehabilitation Nursing, Philadelphia, v. 35, n. 2, p. 47-59, 2010.

WITH, Beverly Palibroda et al. a Practical guide to photovoice: sharing pictures, telling stories and changing communities. Winnipeg: The Prairie Women's Health Centre of Excellence, 2009.

PINI, Maria. Video diaries: questions of authenticity and fabrication. Screening the Past, dez. 2001. Disponível em: $<$ http:// www.screeningthepast.com/2014/12/video-diaries-questions-ofauthenticity-and-fabrication/>. Acesso em: 30 mar. 2018.

PIPER, Heather; FRANKHAM, Jo. Seeing voices and hearing pictures: Image as discourse and the framing of image-based research. Discourse, Abingdon, v. 28, n. 3, p. 373-387, 2007.

PRINS, Esther. Participatory photography: A tool for empowerment or surveillance? Action Research, v. 8, n. 4, p. 426-443, 2010.

PROSSER, Jon. Ìmage-based research: a sourcebook for qualitative researchers. Londres: Falmer Press, 1998. 
PROSSER, Jon; SCHWARTZ, Dona. Photographs within the Sociological Research Process. In: PROSSER, Jon (Ed.). Imagebased research: a sourcebook for qualitative researchers. Londres: Falmer Press, 1998. p. 115-130.

REMILLARD, Chaseten Shynne. Visual representations of homelessness in the canadian public sphere: an analysis of newspaper and photo voice images. 2012. Thesis (Doctor of Philosophy) - University of Calgary, Calgary, Alberta, 2012.

ROSE, Gillian. Visual methodologies. Londres: Sage, 2001.

SINGHAL, Arvind. Empowering the oppressed through participatory theater. Investigación y Desarrollo, v. 12, n. 1, p. 138-163, 2004.

SINGHAL, Arvind et al. Participatory photography as theory, method and praxis: analyzing an entertainment-education project in India1. Critical Arts, v. 21, n. 1, p. 212-227, 2007.

SPIELMAN, Jane. The family photography project: "we will just read what the pictures tell us". Reading Teacher, Newark, Del., v. 54, n. 8, p. 762-770, 2001. 
VAUGHAN, Catherine Maree. A picture of health: participation, photovoice and preventing HIV among papua new guinean youth. 2011. Thesis (Doctor of Philosophy) - School of Economics and Political Science, London 2011.

WALKER, R. Finding a silent voice for the researcher: using photographs in evaluation and research. In: SCHRATZ, Michael (Ed.). Qualitative voices in educational research. Londres: Falmer Press, 1993. Part 2.

WALLERSTEIN, Nina; BERNSTEIN, Edward. Empowerment education: Freire's ideas adapted to health education. Health Education Quarterly, New York, v. 15, p. 379-394, 1988.

WANG, Caroline; BURRIS, Mary Ann. Empowerment through photo novella: portraits of participation. Health Education Quarterly, New York, v. 21, n. 2, p. 171-186, 1994.

WANG, Caroline C. et al. Photovoice as a participatory health promotion strategy. Health Promotion International, Oxford, v. 13, n. 1, p. 75-86, 1998.

WANG, Caroline C. Photovoice: A participatory action research 
strategy applied to women's health. Journal of Women's Healt $h$, v. 8, p. 185-192, 1999.

WANG, Caroline C. et al. Flint photovoice: Community building among youths, adults, and policymakers. American Journal of Public Health, New York, v. 94, n. 6, p. 911-913, 2004.

WANG, Caroline C. Youth participation in photovoice as a strategy for community change. Journal of Community Practice, v. 14, n. 1-2, p. 147-161, 2006.

WANG, Caroline C.; BURRIS, Mary Ann. Photovoice: Concept, methodology, and use for participatory needs assessment. Health Education and Behavior, Thousand Oaks, CA, v. 24, p. 369-387, 1997.

WANG, Caroline C.; BURRIS, Mary Ann; XIANG, Yue Ping. Chinese village women as visual anthropologists: A participatory approach to reaching policymakers. Social Science and Medicine, Oxford, v. 42, p. 1391-1400, 1996.

WANG, Caroline C.; CASH, Jennifer L.; POWERS, Lisa S. Who knows the streets as well as the homeless? Promoting personal 
and community action through photovoice. Health Promotion Practice, v. 1, p. 81-89, 2000.

WANG, Caroline C.; REDWOOD-JONES, Yanique A. Photovoice ethics: perspectives from flint photovoice. Health Education and Behavior, Thousand Oaks, CA, v. 28, p. 560-572, 2001.

WANG, Caroline C.; PIES, C. A. Family, maternal, and child health through photovoice. Maternal and Child Health Journal, v. 8, n. 2, p. 95-102, 2004.

WASHABAUGH, Willian. Philosophical Bases for visual multiculturalism at the college level. In: ELKIN, James (Ed.). Visual literacy. Nova York: Routledge, 2008. p. 129-144.

WILLIAMS, João; LYKES, M. Brinton. Bridging theory and practice: Using reflexive cycles in feminist participatory action research. Feminism \& Psychology, London, v. 13, n. 3, p. 287294, 2003. 